\title{
Characterization and optimization of liquid electrodes for lateral dielectrophoresis $\dagger$
}

\author{
Nicolas Demierre, $\dagger^{*}$ Thomas Braschler, $\$$ Pontus Linderholm, Urban Seger, Harald van Lintel and \\ Philippe Renaud
}

\author{
Received 7th September 2006, Accepted 4th December 2006 \\ First published as an Advance Article on the web 21st December 2006 \\ DOI: $10.1039 / \mathrm{b} 612866 \mathrm{a}$
}

Using the concept of insulator-based "electrodeless" dielectrophoresis, we present a novel geometry for shaping electric fields to achieve lateral deviation of particles in liquid flows. The field is generated by lateral planar metal electrodes and is guided along access channels to the active area in the main channel. The equipotential surfaces at the apertures of the access channels behave as vertical "liquid" electrodes injecting the current into the main channel. The field between a pair of adjacent liquid electrodes generates the lateral dielectrophoretic force necessary for particle manipulation. We use this force for high-speed deviation of particles. By adding a second pair of liquid electrodes, we focus a particle stream. The position of the focused stream can be swept across the channel by adjusting the ratio of the voltages applied to the two pairs. Based on conformal mapping, we provide an analytical model for estimating the potential at the liquid electrodes and the field distribution in the main channel. We show that the simulated particle trajectories agree with observations. Finally, we show that the model can be used to optimize the device geometry in different applications.

\section{Introduction}

In efforts to miniaturize and automatize biological cell handling on microchips, the use of electric fields has received considerable attention, mainly due to the ease and speed of producing electric signals. Dielectrophoresis (DEP) lends itself particularly well to cell handling since comparatively low voltages can produce significant and contactless forces on cells.

DEP is based on the polarizability of particles subjected to an electric field and the subsequent interaction of the induced dipole with the field gradient. ${ }^{1-3}$ The particles are repelled from the regions of high electric field if they are less polarisable than their surrounding medium. In that case, the force is called negative dielectrophoresis (nDEP), while positive DEP occurs if the particles are more polarisable than the medium. nDEP is particularly useful for guiding or trapping cells in flows and can be considered a very gentle cell handling method, as long as excessive electric field strength is avoided. ${ }^{4}$ A typical geometry for deflecting cells consists of facing metal electrodes patterned on the top and the bottom of microchannels. ${ }^{5}$ The fringing of the field at the edges of the electrodes gives rise to $\mathrm{nDEP}$ which is widely used in the field of BioMEMS for trapping, concentrating, separating, deviating, focusing and sorting cells. ${ }^{6-10}$ Coplanar metal electrodes on the bottom of the channel are another typical geometry, used mostly for producing a levitation

Laboratory of Microsystems, Ecole Polytechnique Fédérale de

Lausanne, Lausanne, 1015, Switzerland.

E-mail: nicolas.demierre@epfl.ch; Fax:+41 21693 59 50;

Tel: +41216936838

$\uparrow$ Electronic supplementary information (ESI) available: Calculation of $B, C, D$ and $k$; Matlab file for the calculation of the electric field in a microchannel; Matlab file for the calculation of the cell constant in a microchannel. See DOI: 10.1039/b612866a

\$ The authors have equally contributed to the article. force. Specific applications are field-flow-fractionation (FFF) and traveling wave dielectrophoresis. ${ }^{11-13}$

A more recent development is insulator-based dielectrophoresis (iDEP) also called electrodeless dielectrophoresis ${ }^{14-16}$ (eDEP). The electric field is generally produced by distant current injections, e.g. through platinum wires placed in fluid reservoirs at the periphery of a chip or planar metal electrodes deposited at the extremities of a channel. ${ }^{17,18}$ The distribution of the electric field between the injections is defined by the geometry of the insulator which guides and concentrates the field lines. eDEP avoids some of the major limitations of traditional DEP applications using microelectrodes, which include fouling and destruction of the microelectrodes due to large current densities. Positive eDEP has been used, for example, to trap different species of bacteria or single- or double-stranded DNA between posts ${ }^{19,20}$ and to separate particles according to their size. ${ }^{21}$ The insulator can even be a moving part like an oil droplet ${ }^{22}$ instead of being predefined by a microfabrication process.

Focusing of a particle stream in a microchannel is a prerequisite for a number of applications such as impedance based flow cytometry ${ }^{23}$ or cell sorting and counting. Efficient focusing allows increased throughput and sensitivity. Hydrodynamic focusing is a commonly used method, ${ }^{24,25}$ but it requires supplementary buffer inlets and precise flow control. It is therefore desirable to have a focusing method independent of the flow pattern and fluid control, which motivates the use of DEP-forces for focusing a particle stream. ${ }^{26}$

We present a novel lateral arrangement of patterned metal and insulator for iDEP applications. Large metal electrodes are deposited on the bottom of dead-end chambers positioned perpendicularly to the main channel (Fig. 1a). They are connected to the main channel through constrictions called 


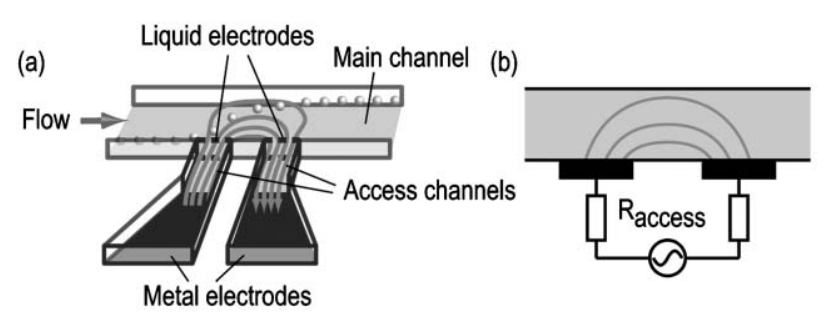

(c)
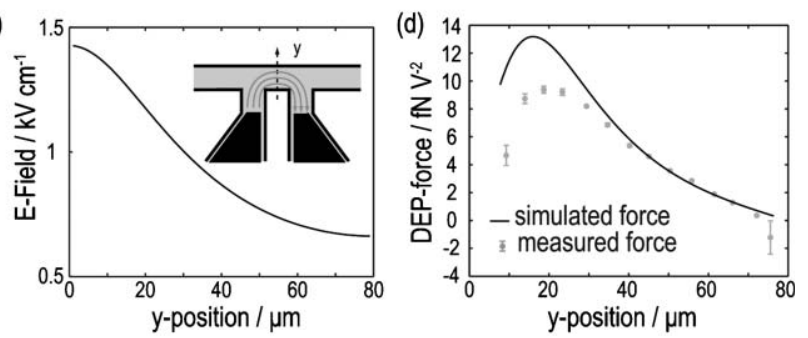

Fig. 1 (a) 3D schematic of the structure for cell manipulation using liquid electrodes. Liquid electrodes refer to the equipotential surface located at the junction of the access and main channels. (b) The model of the liquid electrodes consists of a vertical metal electrode on the sidewall of the main channel with an access resistance. (c) Sample electric field distribution at the symmetry axis of the structure and (d) simulated and measured lateral dielectrophoretic force acting on flowing particles $(5.14 \mu \mathrm{m}$ polystyrene beads with $K(\omega)=-0.5)$ at the symmetry axis (geometrical parameters being listed in Table 1).

access channels and produce, in combination with the insulator, a non-uniform electric field in the main channel. The vertical plane at the boundary of the access and main channel is referred to as "liquid electrode" since it is approximately an equipotential surface. We have developed an analytical model based on conformal mapping to accurately predict the current injection and the potential of the liquid electrode. Liquid electrodes avoid the real estate loss due to reservoirs containing macroscopic electrodes, allowing for potentially massive integration on chip. Nevertheless, the relatively large size of the metal electrodes used for driving the liquid electrodes ensures robustness and high interfacial capacitance, and allows operating nDEP with a broadband signal (from $\mathrm{Hz}$ to $\mathrm{MHz}$ ) at amplitudes smaller than $30 \mathrm{~V}$.

We use the liquid electrodes to deflect trajectories of particles flowing down the main channel. Simulations of trajectories based on the analytical model show good agreement with the measurements. A second application consists of the focusing of a particle stream by adding an opposite dielectrophoretic force with a second pair of liquid electrodes located on the other side of the main channel. The respective magnitudes of the two opposite forces define the position of the focused stream across the channel. The use of two pairs of liquid electrodes located on both sides of the channel allows controlling and reconfiguring the position of the equilibrium by the applied potentials.

\section{Theory}

\section{Liquid electrodes}

The vast majority of BioMEMS using electric fields for actuation or sensing are based on metal microelectrodes. The electrodes are generally patterned on the bottom and/or on the top of a channel in order to take advantage of standard photolithography processes. Since the interfacial capacitance is proportional to the electrode surface, low frequency actuation and sensing is limited by the microelectrodes. One way to avoid these problems in DEP-applications is to use the edge of a comparatively large electrode ${ }^{26}$ at the cost of larger currents and thus potential problems with Joule heating.

We propose here to replace the microelectrodes by distant and large metal electrodes connected to the main channel via comparatively narrow access channels (Fig. 1a). The boundary plane between the access and main channel is approximately an equipotential surface because the field lines are forced to run in parallel by the access channels. The boundary plane therefore has the characteristics of an electrode: defined geometry, defined potential and current injection. Consequently, we refer to this boundary plane between access and main channel as a liquid electrode.

The potential at the liquid electrode is different from the potential applied at the metal electrode lying behind it because of the potential drop across the access channel. Using the technique of conformal mapping (see below), we can estimate both main channel and access channel resistance. The appropriate model for the liquid electrode is therefore a flat vertical electrode located on the main channel wall, characterized by an important but known access resistance, as shown in Fig. 1b. Because of the size of the metal electrode, the double layer impedance of the metal electrode is negligible in the $\mathrm{MHz}$ frequency range used.

The insulator geometry rather than the geometry of the metal electrodes is responsible for the distribution of the field in many respects. Even though the metal electrodes are horizontal and located on the floor of the chip, the current injection into the main channel occurs through the vertical nearly flat equipotential surfaces represented by the liquid electrodes. Moreover, the field distribution is essentially homogeneous across the height of the main channel. Only in the access channel are there important vertical components to the electric field. Since we use the field in the main channel for particle displacements, we essentially observe DEP forces acting in the horizontal plane; hence the term lateral DEP-force.

\section{Dielectrophoresis}

Jones $^{2}$ gives the general formula for the time-averaged DEPforce in the presence of an alternating electric field:

$$
\left\langle\bar{F}_{\mathrm{dep}}\right\rangle=\pi \varepsilon_{\mathrm{m}} r^{3} \operatorname{Re}(\underline{K}(\omega)) \nabla|\bar{E}|^{2}
$$

where $r$ is the particle radius, $\varepsilon_{\mathrm{m}}$ is the permittivity of the medium and $\bar{E}$ is the amplitude of the electric field. $\operatorname{Re}[\underline{K}(\omega)]$ is the real part of the complex Clausius-Mossotti factor. If the particles are approximated by homogeneous spheres, their Clausius-Mossotti factor is given by

$$
\underline{K}(\omega)=\frac{\underline{\varepsilon}_{\mathrm{p}}-\underline{\varepsilon}_{\mathrm{m}}}{\underline{\varepsilon}_{\mathrm{p}}+2 \underline{\varepsilon}_{\mathrm{m}}}
$$

where $\underline{\varepsilon}_{\mathrm{p}}=\varepsilon_{\mathrm{p}}-i \sigma_{\mathrm{p}} / \omega$ and $\underline{\varepsilon}_{\mathrm{p}}=\varepsilon_{\mathrm{m}}-i \sigma_{\mathrm{m}} / \omega . \varepsilon_{\mathrm{p}}, \varepsilon_{\mathrm{m}}$ and $\sigma_{\mathrm{p}}, \sigma_{\mathrm{m}}$ are 
Table 1 List of the geometrical parameters

\begin{tabular}{|c|c|c|c|c|c|c|}
\hline Parameters $^{a}$ & Channel width/ $\mu \mathrm{m}$ & Electrode width $/ \mu \mathrm{m}$ & Insulator width $/ \mu \mathrm{m}$ & Upper voltage/V & Lower voltage/V & Flow speed $/ \mu \mathrm{m} \mathrm{s}^{-1}$ \\
\hline Deviation & 80 & 56 & 56 & - & 27.6 & $\begin{array}{l}\text { (a) }- \text { (b) } 140 \\
\text { (c) } 10 \text { to } 2850\end{array}$ \\
\hline Optimization & $\begin{array}{l}\text { (a)-(b) } 80 \\
\text { (c)-(d) } 20,80\end{array}$ & $\begin{array}{l}\text { (a) } 16,40,56,80,120,160 \\
\text { (b) } 0 \text { to } 160 \\
\text { (c)-(d) } 20,80\end{array}$ & $\begin{array}{l}\text { (a) equal to electrode width } \\
\text { (b) } 0 \text { to } 160 \\
\text { (c)-(d) var. }\end{array}$ & - & 27.6 & $\begin{array}{l}\text { (a)-(b) } 180 \\
\text { (c)-(d) var. }\end{array}$ \\
\hline
\end{tabular}

the permittivities and conductivities of the particles and the medium, respectively, $\omega$ is the angular frequency of the electric field and $i$ is the imaginary unit. We estimated $\operatorname{Re}(\underline{K}(\omega))=-0.496$ for a $2 \mathrm{MHz}$ signal from $\sigma_{\mathrm{m}}, \omega$ and the material data available in the literature: ${ }^{27,28}$

$$
\begin{gathered}
\varepsilon_{\text {medium }}=80 \varepsilon_{0} \\
\varepsilon_{\text {polystyrene }}=3.5 \varepsilon_{0} \\
\sigma_{\text {medium }}=1.4 \mathrm{mS} \mathrm{cm}^{-1}=140 \mathrm{mS} \mathrm{m}^{-1} \\
\sigma_{\text {particle }}=\sigma_{\text {polystyrene }}+\frac{2 K_{\text {surface }}}{r} \approx 0+\frac{2 \times 2 \mathrm{nS}}{5 \times 10^{-6} \mathrm{~m}}=0.8 \mathrm{mS} \mathrm{m}^{-1}
\end{gathered}
$$

We approximate this by taking $\operatorname{Re}(\underline{K}(\omega))=-0.5$ for all calculations. Finally, we need an expression for the electric field in the main channel to calculate the DEP-force.

\section{Calculation of the electric field}

To find an analytical solution to the electric field in the microchannel, we divide the structure into three parts: the main channel and two access channels (Fig. 1a). The electric field and the resistance in the main channel are then calculated, with the hypothesis that the liquid electrodes can be approximated by vertical electrodes located in the main channel wall (Fig. 1b). We subsequently calculate the access resistances and finally the effective voltage between the liquid electrodes by estimating the voltage drop across the access resistances.

We use conformal mapping to transform the physical geometry into a parallel-plate geometry in which the electric field is easily calculated. For a given particular conformal transformation the electric field in the physical plane $E_{z}$ is given as: ${ }^{29,30}$

$$
E_{z}=E_{w} \frac{\overline{\mathrm{d} w(z)}}{\mathrm{d} z}
$$

were $E_{w}$ corresponds to the electric field in the transformed geometry and the second factor is the complex conjugate of the derivative of the transformation. Linderholm et $a .^{31}$ have previously presented a three-step transformation which successively transforms two coplanar electrodes in a microchannel (Fig. 2e) into two parallel electrodes in a simple rectangular geometry (Fig. 2a). Using these three consecutive transformations, the electric field in the microchannel can be found from:

$$
E_{z}=E_{w} \frac{\overline{d w}}{d v} \frac{\overline{d v}}{d u} \frac{\overline{d u}}{d z}
$$

where $w, v$ and $u$ are the three conformal transformations (below). The electric field in the $W$-plane depends only on the effective voltage, which we shall derive later on, and the width of the rectangle and can be written:

$$
E_{w}=\frac{V_{\mathrm{eff}}}{2 K\left(k^{2}\right)}
$$
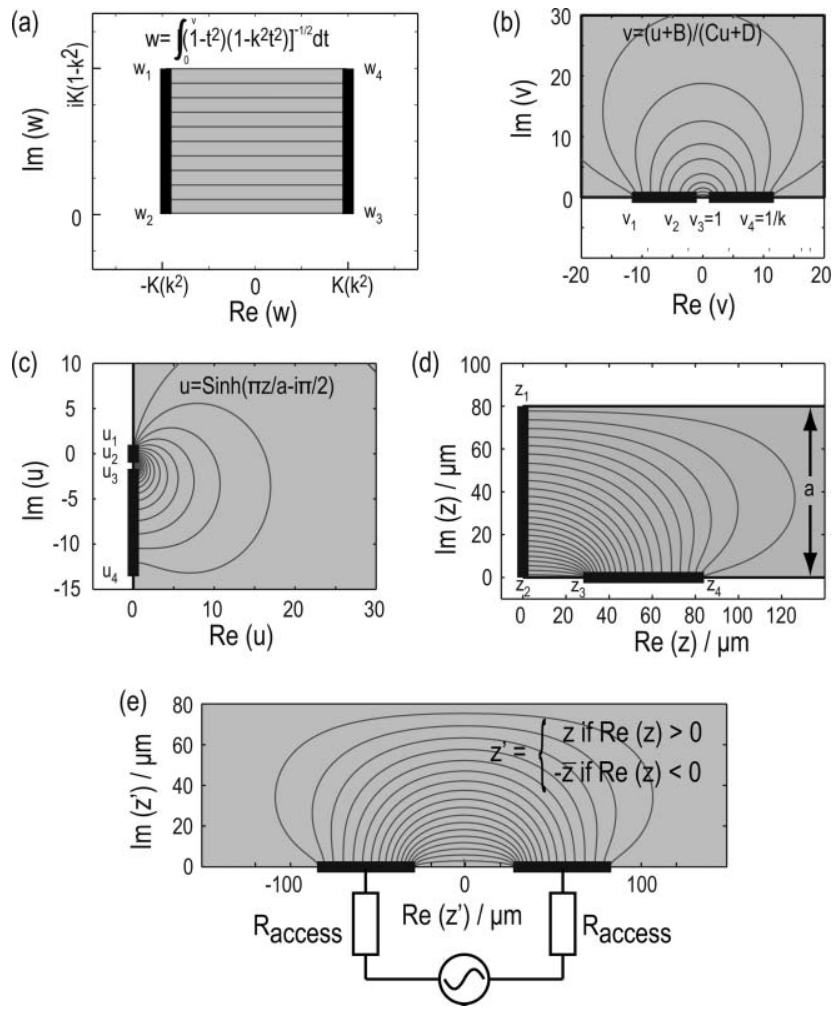

Fig. 2 Successive conformal mappings for obtaining the analytical expression of the electric field between two coplanar electrodes in a sidewall of a microchannel. (a) We start with a simple plate capacitor where $E=V / d$. The electric field strength is also determined by the Schwartz-Christoffel mapping, used to map the half space of (b) into the rectangle in (a). (b) The field distribution in an open half-space with symmetrical arrangement of coplanar electrodes around 0 . The distribution is determined by a bilinear transform from (c) to (b). (c) The field distribution for an asymmetric configuration of electrodes in an open half-plane, which is determined by a complex sinh transform from (d) to (c). (d) The field distribution in rectangular channel geometry. The coordinates in that plane have unit length, since the parameter $a$ describes the channel width. (e) The full field in the structure is obtained by combining the field geometry in twice (d), and taking into account the access resistances. 
where $V_{\text {eff }}$ is the effective voltage and $K\left(k^{2}\right)$ is the complete elliptic integral of the first kind with modulus $k^{2}$.

The Schwartz-Christoffel (S-C) mapping from the $V$-plane (Fig. 2b) to the $W$-plane (Fig. 2a), is defined as:

$$
w(v)=\int_{0}^{v} \frac{\mathrm{d} t}{\sqrt{\left(1-t^{2}\right)\left(1-k^{2} t^{2}\right)}}
$$

where the modulus $k^{2}$ depends only on the channel geometry. Details on the calculation of $k$ are presented in the ESI. $\uparrow$ The derivative of the $\mathrm{S}-\mathrm{C}$ transformation is then:

$$
\frac{\mathrm{d} w}{\mathrm{~d} v}=\frac{1}{\sqrt{\left(1-v^{2}(u(z))\right)\left(1-k^{2} v^{2}(u(z))\right)}}
$$

The second mapping is a bilinear transform, which is used to transform two electrodes of different size into two even-sized electrodes, symmetrically placed around the origin (Fig. 2b). The transformation is written:

$$
v(u)=\frac{u+B}{C u+D}
$$

where $B, C$ and $D$ are real-valued parameters. These parameters result from the constraint that the arrangement of the electrodes in the $v$-plane should be symmetrical around the origin and are also detailed in the ESI. $\uparrow$ The derivative is thus:

$$
\frac{\mathrm{d} v}{\mathrm{~d} u}=\frac{D-B C}{(D+C u(z))^{2}}
$$

The final mapping is a sinh transform, defined as:

$$
u(z)=\sinh \left(\frac{\pi(z-i a / 2)}{a}\right)
$$

where $a$ is the width of the main channel. This transformation folds the rectangular channel geometry, with one electrode being on the dead-end wall of the channel, the other on the lower sidewall (Fig. 2d) into the half-plane $U$ (Fig. 2c). The derivative of the sinh-transform can be written:

$$
\frac{\mathrm{d} u}{\mathrm{~d} z}=\frac{\pi}{a} \cosh \left(\frac{\pi}{a}\left(z-\frac{i a}{2}\right)\right)
$$

Finally, in order to obtain the electric field in a channel with symmetrical electrodes located on one sidewall (Fig. 2e), we use a symmetry argument. The symmetry axis of the structure coincides with an equipotential line, and can thus be replaced by a conducting boundary condition (i.e. an electrode) without altering the field distribution. Therefore, the field distribution of Fig. 2e is obtained by assembling the field distribution of Fig. $2 \mathrm{~d}$ with its mirror image. The electric field $E_{z}{ }^{\prime}$ in the whole structure (Fig. 2e) becomes:

$$
\begin{aligned}
E_{z^{\prime}}= & \frac{1}{2} \frac{V_{\mathrm{eff}}}{2 K\left(k^{2}\right)} \overline{\left(1-v^{2}(u(z))\right)\left(1-k^{2} v^{2}(u(z))\right)^{-1 / 2}} \\
(D-B C) /(D+C u(z))^{2} & \frac{\pi}{a} \cosh \left(\frac{\pi}{a}\left(z-\frac{i a}{2}\right)\right)
\end{aligned}
$$

\section{Effective voltage of the liquid electrodes}

The resistances of the two access channels and the main channel act as a voltage divider, such that the effective voltage difference in the main channel is:

$$
V_{\text {eff }}=V_{0} \frac{R_{\text {channel }}}{R_{\text {channel }}+2 R_{\text {access }}}
$$

where $V_{0}$ is the voltage applied to the metal electrodes. The main channel resistance $R_{\text {channel }}$ is twice the resistance in the $Z$-plane, since we use two elements in series (Fig. 2d and 2e). $R_{z}$, in turn, depends on the cell constant as obtained by the conformal mapping $\left(\kappa_{z}\right)$ and the conductivity of the medium $\sigma_{\mathrm{m}}$ :

$$
R_{\text {channel }}=2 R_{z}=\frac{2}{\sigma_{\mathrm{m}}} \frac{\kappa_{z}}{h}
$$

where $h$ is the height of the channel. Fortunately, the cell constant is an invariant in conformal mappings, so we can use directly the cell constant in the $W$-plane $\kappa_{w}$, which is easy to calculate: ${ }^{32}$

$$
\kappa_{z}=\kappa_{w}=\frac{\left|w_{3}-w_{2}\right|}{\left|w_{1}-w_{2}\right|}=\frac{2 K\left(k^{2}\right)}{K\left(1-k^{2}\right)}
$$

Plugging this expression into eqn (15) yields:

$$
R_{\text {channel }}=\frac{4}{\sigma_{\mathrm{m}} h} \frac{K\left(k^{2}\right)}{K\left(1-k^{2}\right)}
$$

Finally, we calculate the access resistance. Looking at the access channel from the side, it is seen that the metal electrode and the access channel have the geometry shown in Fig. 2d, the metal electrode being the horizontal electrode and the boundary between the main and access channel being the vertical electrode. Therefore, all the mapping steps apply also to the access resistances, albeit obviously with different geometrical factors and parameters $B_{\text {access }}, C_{\text {access }}, D_{\text {access }}$ and $k_{\text {access. }}$ In that way we find the cell constant for the access channel, $\kappa_{\text {access }}$.

The 2D model is based on the hypothesis that the electric field distribution is uniform in the perpendicular direction of the $2 \mathrm{D}$ model. If this is true, the resistance is determined by dividing the cell constant by the length in the perpendicular direction and the medium conductivity (see eqn (15)). However, the distribution of $E_{z}{ }^{\prime}$ across the boundary between main and access channel (Fig. 2e) is not uniform. We need further considerations for estimating $R_{\text {access }}$. The estimation of $R_{\text {access }}$ starts out with Ohm's law:

$R_{\text {access }}=\frac{\langle\Delta V(x)\rangle}{I}=\frac{\Delta V_{\text {access }}}{\int j_{\text {channel }}(x) \mathrm{d} x}=\frac{\Delta V_{\text {access }}}{\sigma_{\mathrm{m}} \int E_{\text {channel }}(x) \mathrm{d} x}$

where $\Delta V_{\text {access }}$ is the average potential drop from the metal electrode to the boundary as seen by the current. The potential drop is not constant, since the boundary between the main and access channel is approximately equipotential, the strict equipotential surface being slightly curved. A weighted average $\Delta V_{\text {access }}$ is estimated from $\Delta V(x)$ and the current density $j_{\text {channel }}(x)$, as obtained from the conformal mapping of the main channel:

$\Delta V_{\text {access }}=\frac{\int j_{\text {channel }}(x) \Delta V(x) \mathrm{d} x}{\int j_{\text {channel }}(x) \mathrm{d} x}=\frac{\int E_{\text {channel }}(x) \Delta V(x) \mathrm{d} x}{\int E_{\text {channel }}(x) \mathrm{d} x}$ 
$\Delta V(x)$, in turn can be calculated from the local current density as obtained from the mapping of the main channel and from the resistance of an element $R_{\mathrm{d} x}$ of width $\mathrm{d} x$ :

$$
\Delta V(x)=\mathrm{d} I R_{\mathrm{d} x}=j_{\text {channel }}(x) \mathrm{d} x R_{\mathrm{d} x}=E_{\text {channel }}(x) \sigma_{\mathrm{m}} \mathrm{d} x R_{\mathrm{d} x}
$$

We can calculate $R_{\mathrm{d} x}$ from the cell constant of the access channel:

$$
R_{\mathrm{d} x}=\frac{\kappa_{\mathrm{access}}}{\sigma_{\mathrm{m}} \mathrm{d} x}
$$

The expression for the access resistance is finally obtained by replacing the $\Delta V_{\text {access }}$ in eqn (18) with eqn (19), (20) and (21):

$$
R_{\text {access }}=\frac{\kappa_{\text {access }}}{\sigma_{\mathrm{m}}} \frac{\int j_{\text {channel }}(x)^{2} \mathrm{~d} x}{\left(\int j_{\text {channel }}(x) \mathrm{d} x\right)^{2}}=\frac{\kappa_{\text {access }}}{\sigma_{\mathrm{m}}} \frac{\int E_{\text {channel }}(x)^{2} \mathrm{~d} x}{\left(\int E_{\text {channel }}(x) \mathrm{d} x\right)^{2}}
$$

The calculation of $R_{\text {access }}$ demonstrates that if we do not consider the correction for a non-uniform electric field distribution, $R_{\text {access }}$ is underestimated by a factor of almost 2. It results in an overestimation of the DEP-force in the main channel. We can now calculate the DEP-force in the main channel from eqn (1) by considering the electric field in eqn (13) and the effective voltage $V_{\text {eff }}$ in eqn (14).

\section{Materials and methods}

\section{Fabrication of the microfluidic device}

Metal electrodes (20 nm Ti for adhesion, $200 \mathrm{~nm} \mathrm{Pt}$ ) were patterned by a lift-off process on $550 \mu \mathrm{m}$ float glass wafers. Channels were then produced on top of the electrodes by structuring a $20 \mu \mathrm{m}$ layer of SU-8. The whole structure was reversibly sealed by a flat piece of PDMS containing the access holes. The wafer was diced into $20 \times 15 \mathrm{~mm}$ chips. The chips were mounted into custom-made fluidic interface including liquid reservoirs and connected to a printed circuit board with an electric interface based on spring contacts. The PDMS piece and the chip were treated by oxygen plasma (40 s at $50 \mathrm{~W}$ ) prior to use for making them temporarily hydrophilic and therefore enhancing the priming. This was especially important to allow priming for the electrode chambers.

Fluorescent beads (polystyrene, carboxyl-modified, $5.14 \mu \mathrm{m}$ diameter) were used for tracking the particle trajectories and as models for evaluating the performance of the devices. In all experiments, we used diluted PBS with a conductivity of $1.4 \mathrm{mS} \mathrm{cm}^{-1}$, as measured by the conductivity meter Cyberscan CON100.

The electrodes were driven by signals at $2 \mathrm{MHz}$ from an $\mathrm{HP}$ 33120A signal generator and amplified by custom electronic circuits to produce signals with a maximum amplitude of $27.6 \mathrm{~V}$

\section{Particle tracking}

Particle trajectories were obtained by video recordings of the fluorescence of the beads. The particle tracking was then performed in two steps using sequential image analysis in VirtualDub and ImageJ. In the first step, we used VirtualDub, an open source video filtering program, to convert the video recording into a sequence of grey frames. We then applied a threshold to each image for extracting the information of interest. At high speeds, the particles tend to appear as interrupted lines due to the $100 \mathrm{~Hz}$ discharge of the mercury arc lamp. A longitudinal blur corrects this effect, i.e. a convolution with an ellipsoidal point spread function, before thresholding. This blurs out the particles along the axis of motion, but the center of gravity is conserved and it avoids the appearance of spurious supplementary particles. In the second step, we loaded the frame sequences into ImageJ as stacks and processed them with the open source plugin Mtrack2. ImageJ was chosen because of the liberty given to the user in programming or customizing plugins. We customized Mtrack2 in order to account for general laminar flow. We modified the definition of the distance traveled by a given particle from one frame to the next by subtracting the displacement due to the mean flow speed from the total displacement of the particle. The Mtrack2 produces a list of particle positions in different frames as its output. Speed vectors and particle positions were finally calculated in the commercial software Matlab./

\section{Simulation of particle trajectories}

We used the analytical expression of the DEP-force (eqn (1), (13) and (14)) to simulate the trajectory of a particle flowing through the channel. Both $R_{\text {access }}$ and $R_{\text {channel }}$ were calculated from the channel geometry and fluid conductivity (eqn (15) and (22)). As we work in microchannels with low Reynolds numbers ( 0.01 to 0.1 with standard experimental conditions), inertial forces are negligible compared to viscous forces and the particle motion is defined by the equilibrium between DEP-force and drag force as: ${ }^{33}$

$$
6 \pi \eta r\left(v-v_{\text {flow }}\right)=F_{\text {DEP }}
$$

where $r$ and $v$ are the particle radius and the velocity and $\eta$ is the viscosity of the medium. The velocity of the particle during a time interval $\mathrm{d} t$ is assumed constant and equal to the terminal velocity:

$$
v=\frac{F_{\mathrm{DEP}}}{6 \pi \eta r}+v_{\text {flow }}=\frac{\pi \varepsilon_{\mathrm{m}} r^{3}(-0.5) \nabla|E|^{2}}{6 \pi \eta r}+v_{\text {flow }}
$$

where we have plugged in $\underline{K}(\omega)=-0.5$, as explained above.

In the simulations, we assume homogeneous speed in the $x$-direction and no flow in the $y$-direction at all locations in the channel. Particle trajectories are then obtained by calculating the displacement of the particles during short time intervals. We chose a $\mathrm{d} t$ sufficiently short such that $v \mathrm{~d} t<1 \%$ of the channel width in all cases, but on some figures we report particle positions at larger spacings for reasons of visibility.

The deviation was defined as the lateral displacement of a particular particle entering the channel along the lower wall. This corresponds to the maximum displacement that the structure can impart on a particle at a given general flow speed. 


\section{Results and discussion}

\section{DEP-force}

Obtaining the DEP-force from particle trajectories is generally complicated, as DEP-force and fluid flow are not at right angles. A commonly employed method is stopped flow, but this turned out to be problematic because of flow control and bead sedimentation, making it tedious to obtain forces along a given line in the structure. However, by simple symmetry reasoning, one understands that fluid flow at the symmetry axis (see inset of Fig. 1c) must only have an $x$-component which agrees with the basic reversibility in laminar flow. By a similar argument, it is also clear that the electric field gradient has no $x$-component at the symmetry axis of the structure. Consequently the fluid flow at the symmetry axis is only in the $x$-direction while the DEP-force is only in the $y$-direction.

Using a simple structure consisting of a pair of adjacent liquid electrodes located in the channel sidewall, we quantified the DEP-force at the symmetry axis acting on particles flowing down the main channel by measuring the $y$-component of the speed at the symmetry axis and applying eqn (23). Table 1 lists the geometrical dimensions of the structure and the experimental parameters. Fig. 1d compares the DEP-force estimated from particle trajectories to the analytical calculation. The general shape of the curves is similar and at distances far away from the electrodes (the upper sidewall of the channel corresponding to the right hand side of the graph) the agreement is excellent. Note that the force is expressed in $\mathrm{fN} \mathrm{V}^{-2}$ because of the normalization. A typical voltage of $25 \mathrm{~V}$ therefore gives rise to a DEP-force on the order of $5 \mathrm{pN}$ on the beads.

Close to the electrodes, the measured force is smaller than the calculated. This can be attributed to soft fringing: the orifice of the liquid electrodes acts only approximately like a metal electrode. More specifically, the equipotential surface defining the opening is not flat, but curved such that the field gradients close to liquid electrodes are somewhat less pronounced than for metal electrodes. This may be advantageous

(a)

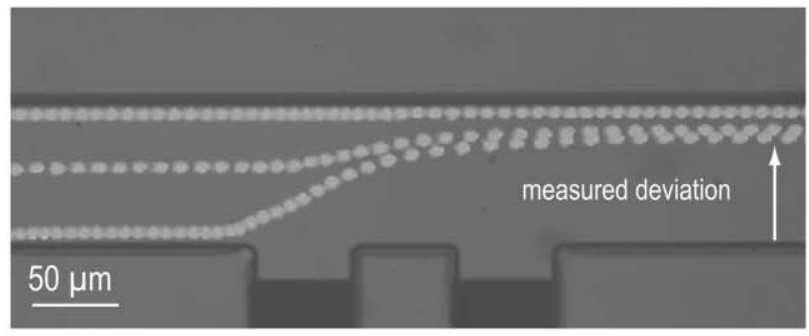

(b)

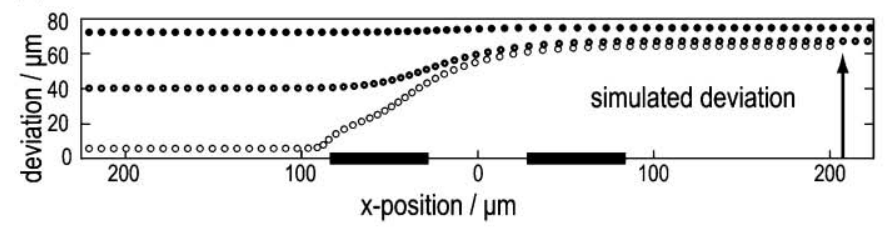

for biological applications, as very strong fields can cause cell damage and protein denaturation.

The relative error of the measurement close to the electrodes is rather big since we had to lower the electric field strength to avoid strong particle repulsion from this region. On the other side of the channel, the last point of measurement reveals a negative value for the DEP-force. This means that most of the measured trajectories at this location have a negative $y$-component. This effect is probably caused by locally enhanced field non-uniformities between the insulating particles and the insulating channel wall. The error of the measurement at this position is also important. It comes from the $y$-component of the particle trajectories that can either be negative (very close to the wall) or positive (somewhat further away).

\section{Deviation}

Using the same structure, we quantified the displacement of particles caused by the electric field by recording and analyzing time-lapse videos. An overlay of video stills extracted from such a time-lapse experiment is shown in Fig. 3a. Fig. 3b shows simulated particle trajectories for a set of parameters similar to the ones used in the video recording in Fig. 3a which are listed in Table 1. Visual comparison shows good agreement of the shape of the particle trajectories and also of the total displacement towards the upper part of the main channel experienced by the particles.

To obtain a quantitative result, the deviation at various speeds was analyzed (Fig. 3c). The deviation was defined as the width of the particle-free zone in the lower part of the channel downstream of the liquid electrodes. Comparison of theoretically determined and experimentally observed deviation in Fig. 3c shows good agreement. The measured values and the errors are based on classical statistics.

The predictions of displacement by simulation could be further improved if we could better evaluate the particle speed. We observed that the speed is frequently increased in the downstream region of the structure. These increases in the

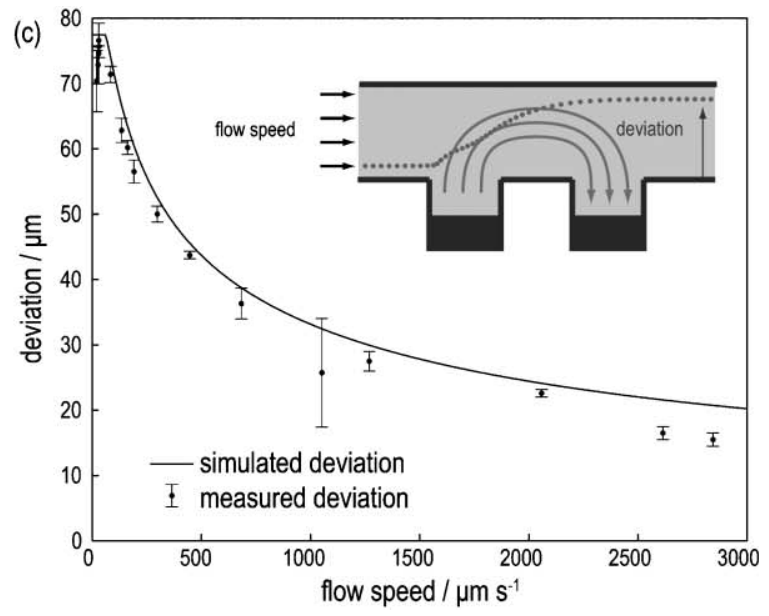

Fig. 3 Particle tracking of lateral deviation. (a) Overlay of video stills taken at a rate of $75 \mathrm{fps}$, with a fluid flow speed of $140 \mu \mathrm{m} \mathrm{s}{ }^{-1}$. (b) Numerical simulation of lateral deviation with identical geometry and parameters as used in (a). (c) Lateral deviation as a function of flow speed in the same structure, both measured (points) and simulated (solid line). Table 1 lists the geometrical parameters. For each simulation, a constant flow speed with speed vectors aligned along the $x$-axis is assumed, as well as $\underline{K}(\omega)=-0.5$ for the $5.14 \mu \mathrm{m}$ beads. 
speeds are likely due to a vertical centering of particles in the main channel. Indeed, an insulating particle located at an insulating boundary squeezes the electric field lines, which locally creates an electric field non-uniformity. This repels the particles from the insulator.

\section{Focusing}

We propose here a combination of two pairs of liquid electrodes, located on opposite sides of the main channel, to achieve the focusing of a stream of beads (Fig. 4a). Each pair is individually able to deviate the particles towards the opposite sidewall. The combined action of the two pairs focuses the particle stream towards an equilibrium position. The structure we used in this experiment has a channel width of $20 \mu \mathrm{m}$; the other parameters being listed in Table 1.

(a)
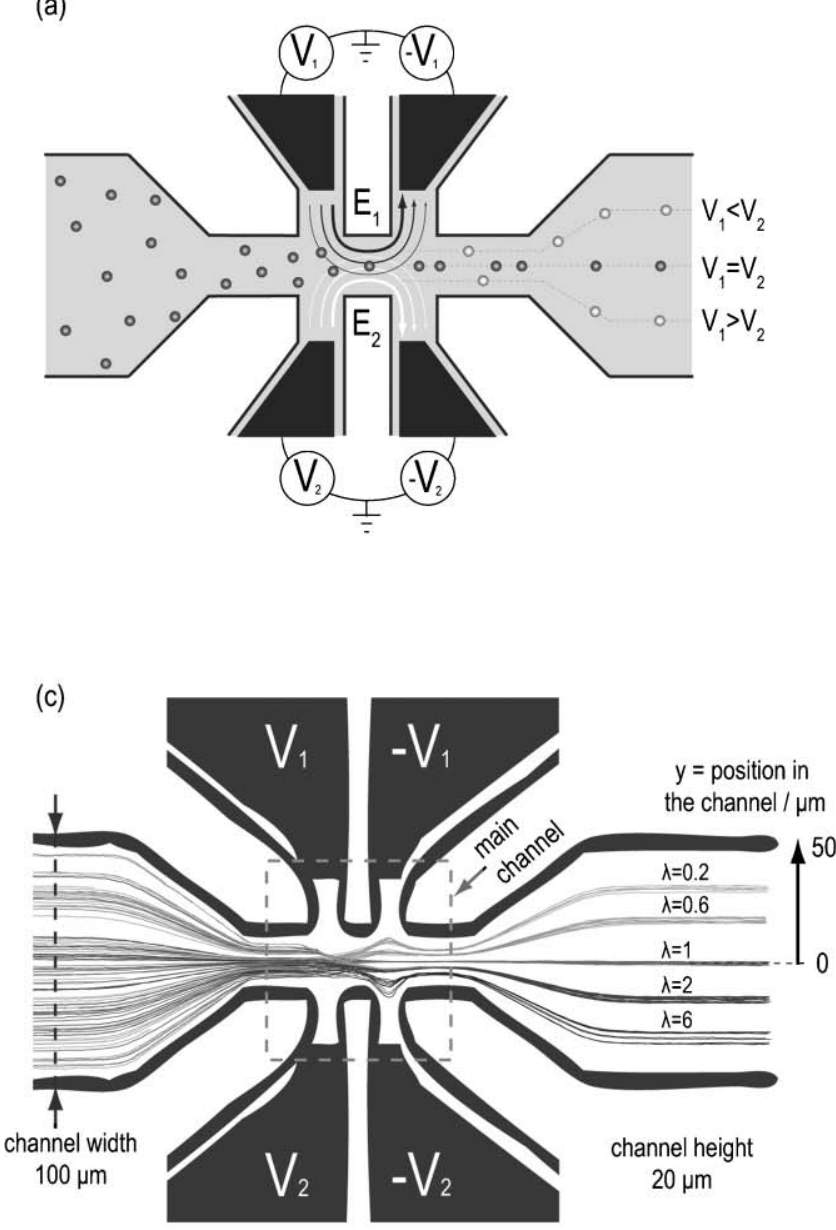

Two electric fields $E_{1}$ and $E_{2}$ are generated in the main channel giving rise to two opposing DEP-forces. The magnitudes of the forces are directly controlled by the applied potentials $V_{1}$ and $V_{2}$. The tuning of the focusing position in the channel is controlled by the ratio of the two potentials $V_{1} / V_{2}$. If the lower potential is larger than the upper potential, the particles are focused towards a position in the upper half of the channel and vice-versa. Since the DEP-force is determined by the gradient of the square of the magnitude of the electric field (eqn (1)), it also depends on the phase shift between $\bar{E}_{1}$ and $\bar{E}_{2}$. If the phase shift is $\pi / 2$, then $\bar{E}^{2}=\bar{E}_{1}{ }^{2}+\bar{E}_{2}{ }^{2}$ and the total force is just the sum of the individual forces, $\bar{F}_{\text {tot }}=\bar{F}_{1}+\bar{F}_{2}$. It also turns out experimentally that at $\pi / 2$ phase shift there is the strongest focusing with the least trapping effect, so we generally used $\pi / 2$ out of phase signals for $V_{1}$ and $V_{2}$.

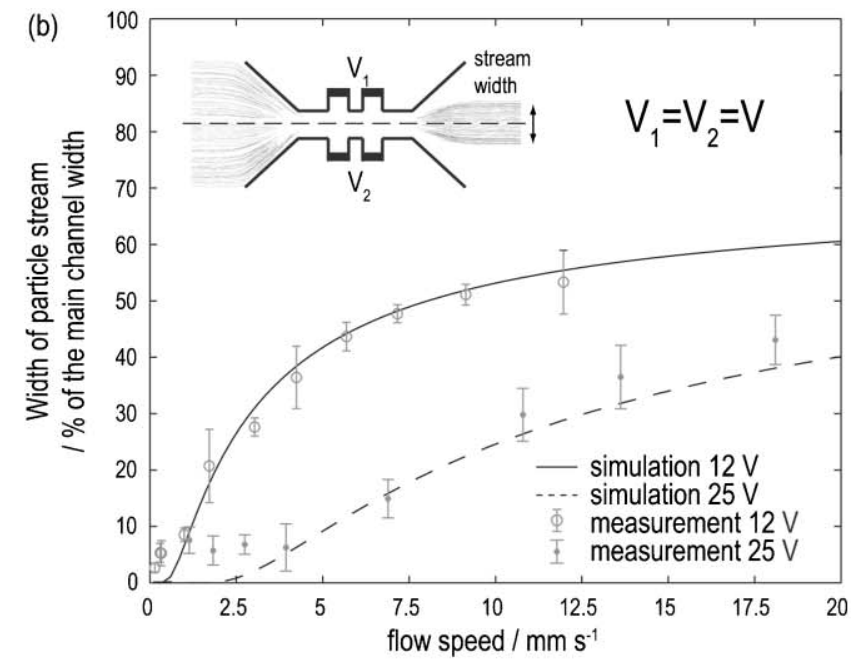

(d)

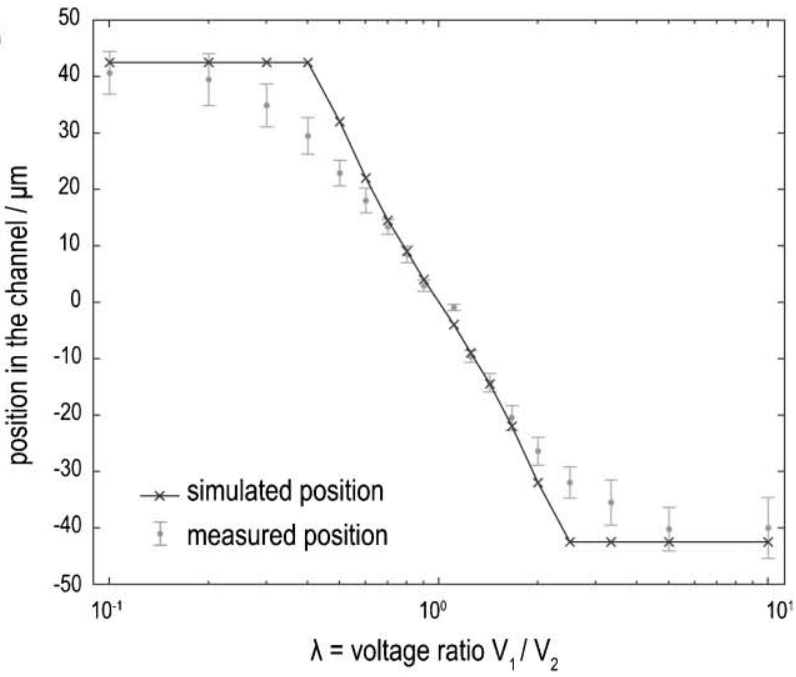

Fig. 4 Focusing of a particle stream. (a) A four liquid electrodes structure. Particles flowing from left to right are deflected by forces produced by both electric fields $E_{1}$ and $E_{2}$. The position of focusing can be shifted in the $y$-direction by adjusting the ratio $\lambda=V_{1} / V_{2}$. (b) Midline focusing of a particle stream for $V_{1}=V_{2}$. We evaluated the focusing performance as a function of speed at two voltages. All the geometrical and electrical parameters are listed in Table 1. The fields act as a barrier below a minimum speed and accumulate particles in front of the structure. Above this critical speed, particles are focused precisely to the middle of the channel. Above a second threshold speed (about $0.5 \mathrm{~mm} \mathrm{~s}{ }^{-1}$ for $12 \mathrm{~V}$ and about $5 \mathrm{~mm} \mathrm{~s}^{-1}$ for $25 \mathrm{~V}$ ), focusing imperfection exceeds the basal influence of particle collision, so that the stream is widening. (c) Shift of the position of the particle stream from one side to the other side as a function of $\lambda$. We show 5 sets of traces obtained for 5 different $\lambda$. (d) The quantitative dependence of the focusing position on $\lambda$ for the simulated positions (black crosses in solid line, simulated at the symmetry axis and reported after the structure) and for the measured positions (grey points, measured after the structure). 
We first investigated the performances of the particle focusing towards the midline of the structure at different flow speeds. We obtained a focusing in the middle of the channel by applying equal voltages $V_{1}$ and $V_{2}$. Fig. $4 \mathrm{~b}$ shows measurements and simulations of the evaluation of the focusing in term of percentage of the channel occupied by the particle stream as a function of the flow speed for two voltages. Below a minimum speed $\left(0.1 \mathrm{~mm} \mathrm{~s}^{-1}\right.$ for $12 \mathrm{~V}, 1 \mathrm{~mm} \mathrm{~s}^{-1}$ for $\left.25 \mathrm{~V}\right)$, the fields act as a barrier and there is accumulation of particles in front of the structure. Above this speed, particles are focused precisely to the middle of the channel. Due to particle diffusion and collisions, we can never observe $0 \%$ particle stream width. Above a second threshold speed (about $0.5 \mathrm{~mm} \mathrm{~s}^{-1}$ for $12 \mathrm{~V}$ and about $5 \mathrm{~mm} \mathrm{~s}^{-1}$ for $25 \mathrm{~V}$ ), the stream starts widening. The evolution of the widening of the stream width is well predicted by the simulations. The stream width is a non-linear function of the speed due to the strongly non-linear distribution of the DEP-forces across the channel (see Fig. 1d). However, up to very high speeds (several $\mathrm{mm} \mathrm{s}^{-1}$ ), the particle stream remains focused to an appreciable extent.

Finally, we demonstrated the focusing towards variable positions in the main channel as a function of the ratio of the applied voltages $\lambda=V_{1} / V_{2}$ at constant flow speed of $2.7 \mathrm{~mm} \mathrm{~s}^{-1}$. Fig. 4c is a superposition of a picture of the structure and the particle traces as obtained by particle tracking at sample voltage ratios $\lambda$. The deviation of the particles towards the lower part of the channel is obtained by keeping $V_{1}$ constant at $25 \mathrm{~V}$ while varying $V_{2}$ from $2.5 \mathrm{~V}$ to $25 \mathrm{~V}$ and inversely for the focusing in the upper part of the channel. The two DEP-forces define an equilibrium line where the two forces have equal and opposite $y$-components. Varying $\lambda$ shifts this equilibrium line vertically in the main channel; and since particles continue to be focused, this means that the focused particle stream after the structure is vertically displaced across the main channel width. We can approximately calculate the position of the equilibrium line by examining the force profile at the vertical symmetry axis. By setting the DEP-forces from both sides equal, we obtain an equilibrium position on the vertical symmetry axis.

The calculated positions of the equilibrium and the measured values as a function of $\lambda$ are compared in Fig. $4 d$. The measured position is the mean value of the $y$-position of the beads after the focusing element, while the error estimation is twice the standard deviation of the position. In general the agreement between theory and measurement is good, although the particle stream is closer to the midline than calculated for small and big $\lambda$. The theory predicts that the particles should be completely pushed towards the sidewall of the weaker voltage for $\lambda$ smaller than 0.4 and bigger than 2.5 . This would result in particles entering the access channels. However, we observed that the particles were pushed into the electrode chambers only for $\lambda$ smaller than 0.1 and bigger than 10 . Most likely the field concentrations around the corners of the access channel openings keep particles from entering the electrode chambers. This effect is not taken into account by our estimation of the equilibrium position at the symmetry axis of the structure. In addition, it is seen that the standard deviation of the position increases as $\lambda$ deviates from 1 . Since we keep the higher voltage constant, $\lambda$ values far from 1 signify a very weak opposing force and also larger displacements necessary for some of the particles to reach their equilibrium position, meaning that the flow speed was somewhat too fast for these combinations, while it was well chosen for $\lambda$ values closer to 1 . The deviation between the simulation and the measurement also comes from the fact that the equilibrium positions are calculated at the symmetry axis of the structure, whereas the measurement of the positions are done after the structure. In that case, the electric field non-uniformities acting after the symmetry axis are not considered in the calculation. Despite these minor deviations, the simulations, as well as the measurements, demonstrate the possibility of focusing a particle stream at a desired position everywhere in the main channel on an equilibrium line. This four-electrode structure presents the great advantage that the distribution of the force and thus the position of the equilibrium line can be easily tuned by adjusting the potentials applied to the pairs of metal electrodes and therefore the liquid electrodes. The experiments presented in this section show the ability of this structure to efficiently focus particle streams with very high speed as well as the ability to position the particle stream anywhere in the channel. These are two important points in many microfluidic applications.

\section{Optimization of the design}

Having demonstrated the accuracy of our model in predicting experimental outcome, we shall now use it to propose some design considerations. We modified some geometrical dimensions of the structure used in Fig. 3 and evaluated the effects on the deviation by measurements and simulations. In Fig. 5a we simultaneously varied electrode width and insulator width from 16 to $160 \mu \mathrm{m}$ (see Table 1). Fig. 5a shows the measurements of deviation based on these different geometries. We observed excellent agreement of the measured and the simulated deviation. Our analytical model for the liquid electrode based DEP therefore works also in varying geometries.

We then extended the analysis to independent variation of electrode width and insulator width. This gives rise to a bidimensional parameter space, where the lateral deviation of the particle stream at a given flow speed and voltage is the variable to optimize. The $3 \mathrm{D}$ Fig. $5 \mathrm{~b}$ gives the distribution of the deviation in this parameter space. The design should avoid the regions with too small electrode or insulator width, while there is a relatively large zone in the parameter space that produces acceptable results. There is an optimum for the insulator width at a given electrode width, while for a given insulator width, the larger the electrodes, the more efficient the DEP deviation. Fig. 5b intends to give a feeling about the system, while Fig. 5c and d should enable engineers to do design work. In the following section, we outline some design rules based on these simulation results.

As a general rule, for a given applied voltage, smaller channels induce larger electric field and larger field non uniformity. Therefore, we should design the channels as small as possible regarding the particle size. The coupling capacitance between the metal electrode and the liquid is not an issue since the metal electrodes are distant and can be much bigger 

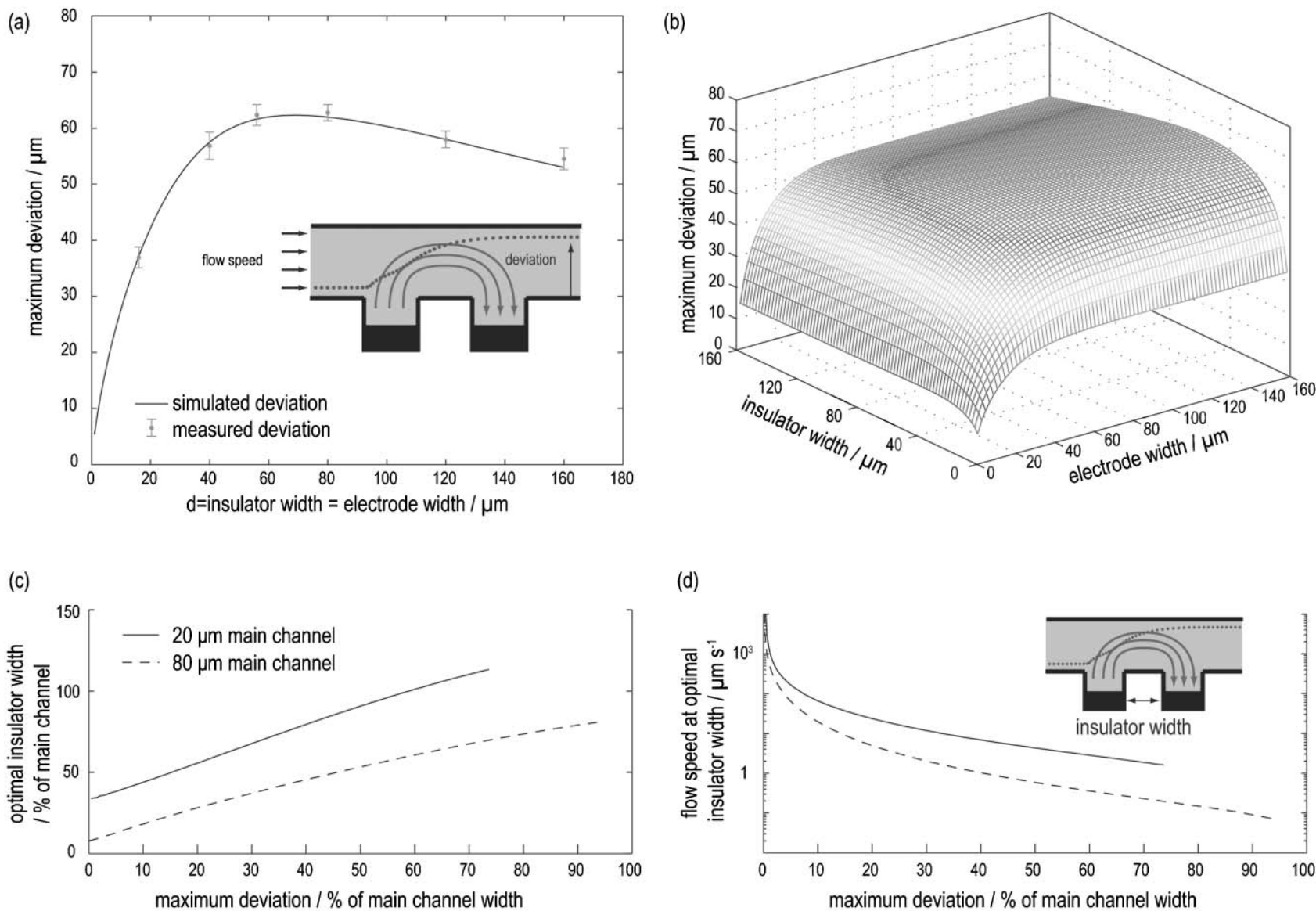

Fig. 5 Design optimization. This figure describes the influence of three geometrical parameters on the performance of the deviation: main channel width, electrode width and insulator width. Device performance is characterized by how much beads initially flowing on the side of the electrodes are deviated towards the other side of the channel, which represents the maximum deviation. In (a), electrode width and the insulator width are both set to a common value $d$, which varies from $16 \mu \mathrm{m}$ to $160 \mu \mathrm{m}$. There is an optimum for the design parameter $d$, at around $70 \mu \mathrm{m}$. The other parameters are listed in Table 1. (b) This is a numerical exploration of the bi-dimensional parameter space provided by the independent variation of electrode width and the insulator width, the other parameters being identical to (a). At a given electrode width, there is an optimum for the insulator width. For a given insulator width, on the contrary, the larger the electrode, the more the beads are deviated. Still, once the electrode width exceeds the width of the main channel (here $80 \mu \mathrm{m}$ ), there is only minimal improvement in device performance. In (c), we determined the optimal insulator width as a function of desired deviation across the main channel by numerical simulation of particle trajectories by repeated steps of simulation and optimization. The optimizations were carried out for 180 different speeds, both for a $20 \mu \mathrm{m}$ and $80 \mu \mathrm{m}$ main channel width, looking each time for the insulator width producing the maximum deviation. In all cases, the electrode width was set equal to the main channel width. (d) Reports the maximum flow speeds at optimal insulator width and at $27.6 \mathrm{~V}$.

than the characteristic size of the structures. As shown in Fig. $5 \mathrm{~b}$, the wider the electrodes are, the bigger the particle deviation is. However, large electrode width has also a major fluidic drawback, since the laminar flow will extend into the electrodes. This could lead to deposition of particles on the electrodes and to trapping effects at the electrode edges for inert particles or more seriously to destruction of sensitive particles such as cells in the immediate vicinity of the metal electrodes. We found that making the electrode width equal to the main channel width is a good rule of thumb; at this size, DEP deviation is almost maximal, while flow into and out of the electrode chambers is still relatively limited. Then Fig. 5c helps to choose the optimal insulator width for the DEP application as a function of the desired maximum deviation in term of the percentage of the main channel width. Finally, Fig. $5 \mathrm{~d}$ indicates the maximum flow speed in order to achieve the desired deviation at the optimal insulator width.
In the case of downscaling all dimensions (including photoresist thickness), the electric field conserves its shape and Fig. 5c remains valid. Fig. 5d obviously depends on the applied voltage, and higher or lower voltage would shift the curve vertically toward higher or lower maximum flow speed. Note that the finite size of the beads avoids achieving $100 \%$ of deviation.

\section{Summary and conclusion}

We have shown that dielectric particles can accurately be positioned in a microchannel by using DEP-forces generated by the electric field between so-called liquid electrodes. Compared to wire electrodes placed in reservoirs, the proposed solution allows for a more compact integration. Compared to top and bottom microelectrodes, planar electrodes are significantly easier to fabricate since no bonding and no 
alignment are required. In addition, the use of large metal electrodes in separate chambers enables higher current densities and a longer electrode lifetime. Furthermore, the large size of the metal electrode implies a large interfacial capacitance. This results in lower impedance, extending the frequency range of operation to lower frequencies.

According to the principles of electrodeless DEP, the nonuniformity of the electric field in the main channel is no longer dependent on the metal electrode geometry but rather on the arrangement of the main and access channels, i.e. the insulator geometry. The term "liquid electrode" refers to a vertical equipotential surface located at the junction of the main and access channels. The model we propose analytically defines the current injection and the potential at the liquid electrodes and shows excellent agreements when compared to measurements, as estimated by DEP-forces and particle deviations generated by the devices. The calculation of the electric field is based on conformal mapping steps, which transform a rectangular geometry with facing electrodes, i.e. a plate capacitor, into a channel geometry with planar electrodes. Although in this paper we make use of the mapping to evaluate the field between two adjacent planar electrodes, the placement of the electrodes is arbitrary. So, with few adaptations, the mapping steps described above can also be used to suit other needs, such as the calculation of the field between electrodes on opposite sides of the channel. Typical applications include modeling impedance measurements and deviation of particles by facing microelectrodes.

A first example of the possibilities offered by this concept is the ability to focus particles to an arbitrary position in the channel using a unique four-electrode structure. The position of the equilibrium between two facing DEP-forces is controlled and reconfigurable by adjusting the applied voltages. Since the total force can be modeled accurately by superimposing the two force fields, this means that the liquid electrodes indeed behave as independent entities.

The technology presented is not limited to DEP-manipulation, but could also be extended to impedance measurements of dielectric particles such as cells, especially at lower frequencies where the large interfacial capacitance becomes important. Since the interfacial capacitance of the liquid electrodes is very large and independent of its resistance, the concept could also be downscaled to measure and manipulate nanoparticles such as viruses and macromolecules.

\section{Acknowledgements}

The authors gratefully thank Dr Arnaud Bertsch, Valentin and Adelina Simeonov, and André Mercanzini for reading the manuscript. This work was supported by the European Community-funded CellPROM project under the 6th Framework Programme, contract No. NMP4-CT-2004500039 .

\section{References}

1 Y. Huang and R. Pethig, Electrode design for negative dielectrophoresis, Meas. Sci. Technol., 1991, 2(12), 1142-1146.

2 T. B. Jones, Electromagnetics of Particles, Cambridge University Press, Cambridge, 1995.
3 H. A. Pohl, Dielectrophoresis, Cambridge University Press, London, 1978.

4 A. Menachery and R. Pethig, Controlling cell destruction using dielectrophoretic forces, IEE Proc., Nanobiotechnol., 2005, 152(4), $145-149$.

5 M. Dürr, J. Kentsch, T. Müller, T. Schnelle and M. Stelzle, Microdevices for manipulation and accumulation of micro- and nanoparticles by dielectrophoresis, Electrophoresis, 2003, 24(4), $722-731$.

6 P. R. C. Gascoyne and J. Vykoukal, Particle separation by dielectrophoresis, Electrophoresis, 2002, 23(13), 1973-1983.

7 M. P. Hughes, Strategies for dielectrophoretic separation in laboratory-on-a-chip systems, Electrophoresis, 2002, 23(16), 2569-2582.

8 T. Müller, G. Gradl, S. Howitz, S. Shirley, T. Schnelle and G. Fuhr, A 3-D microelectrode system for handling and caging single cells and particles, Biosens. Bioelectron., 1999, 14(3), 247-256.

9 S. Fiedler, S. G. Shirley, T. Schnelle and G. Fuhr, Dielectrophoretic Sorting of Particles and Cells in a Microsystem, Anal. Chem., 1998, 70(9), 1909-1915.

10 U. Seger, S. Gawad, R. Johann, A. Bertsch and P. Renaud, Cell immersion and cell dipping in microfluidic devices, Lab Chip, 2004, 4(2), 148-151.

11 J. G. Kralj, M. T. W. Lis, M. A. Schmidt and K. F. Jensen, Continuous dielectrophoretic size-based particle sorting, Anal. Chem., 2006, 78(14), 5019-5025.

12 X.-B. Wang, J. Yang, Y. Huang, J. Vykoukal, F. F. Becker and P. R. C. Gascoyne, Cell separation by dielectrophoretic field-flowfractionation, Anal. Chem., 2000, 72(4), 832-839.

13 G. Fuhr, R. Hagedorn, T. Muller, B. Wagner and W. Benecke, Linear motion of dielectric particles and living cells in microfabricated structures induced by traveling electric fields, Proc. IEEE Microelectromech. Syst., 1991.

14 C.-F. Chou and F. Zenhausern, Electrodeless Dielectrophoresis for Micro Total Analysis Systems, IEEE Eng. Med. Biol. Mag., 2003, 22(6), 62-67.

15 L. M. Barrett, A. J. Skulan, A. K. Singh, E. B. Cummings and G. J. Fiechtner, Dielectrophoretic manipulation of particles and cells using insulating ridges in faceted prism microchannels, Anal. Chem., 2005, 77(21), 6798-6804.

16 S. Masuda, M. Washizu and T. Nanba, Novel method of cell fusion in field constriction area in fluid integrated circuit, IEEE Trans. Ind. Appl., 1989, 25(4), 732-737.

17 Y. Huang, N. Chen, J. Borninski and B. Rubinsky, A novel microfluidic cell-chip for single cell analysis and manipulation, Proc. IEEE Microelectromech. Syst., 2003.

18 H. Chun, T. D. Chung and H. C. Kim, Cytometry and velocimetry on a microfluidic chip using polyelectrolytic salt bridges, Anal. Chem., 2005, 77(8), 2490-2495.

19 C.-F. Chou, J. O. Tegenfeldt, O. Bakajin, S. S. Chan, E. C. Cox, N. Darnton, T. Duke and R. H. Austin, Electrodeless dielectrophoresis of single- and double-stranded DNA, Biophys. J., 2002, 83(4), 2170-2179.

20 B. H. Lapizco-Encinas, B. A. Simmons, E. B. Cummings and Y. Fintschenko, Dielectrophoretic Concentration and Separation of Live and Dead Bacteria in an Array of Insulators, Anal. Chem., 2004, 76(6), 1571-1579.

21 K. H. Kang, Y. Kang, X. Xuan and D. Li, Continuous separation of microparticles by size with direct current-dialectrophoresis, Electrophoresis, 2006, 27(3), 694-702.

22 I. Barbulovic-Nad, X. Xuan, J. S. H. Lee and D. Li, DCdielectrophoretic separation of microparticles using an oil droplet obstacle, Lab Chip, 2006, 6(2), 274-279.

23 S. Gawad, L. Schild and P. Renaud, Micromachined impedance spectroscopy flow cytometer for cell analysis and particle sizing, Lab Chip, 2001, 1(1), 76-82.

24 G.-B. Lee, C.-I. Hung, B.-J. Ke, G.-R. Huang, B.-H. Hwei and H.-F. Lai, Hydrodynamic focusing for a micromachined flow cytometer, J. Fluids Eng., 2001, 123(3), 672-679.

25 C. C. Chen, S. Zappe, O. Sahin, X. J. Zhang, M. Fish, M. Scott and O. Solgaard, Design and operation of a microfluidic sorter for Drosophila embryos, Sens. Actuators, B, 2004, 102(1), 59-66.

26 D. Holmes, H. Morgan and N. G. Green, High throughput particle analysis: Combining dielectrophoretic particle 
focussing with confocal optical detection, Biosens. Bioelectron., 2006, 21(8), 1621-1630.

27 W. M. Arnold, H. P. Schwan and U. Zimmermann, Surface Conductance and Other Properties of Latex-Particles Measured by Electrorotation, J. Phys. Chem., 1987, 91(19), 5093-5098.

28 X.-F. Zhou, G. H. Markx, R. Pethig and I. M. Eastwood, Differentiation of viable and non-viable bacterial biofilms using electrorotation, Biochim. Biophys. Acta, 1995, 1245(1), 85-93.

29 L. J. Binns, P. J. Lawrenson and C. W. Trowbridge, The Analytical and Numerical Solution of Electric and Magnetic Fields, John Wiley \& Sons, 1992.
30 R. Schinzinger and P. A. A. Laura, Conformal mapping: methods and applications, Elsevier, Amsterdam, 1991.

31 P. Linderholm, P. Renaud, J. Hong and D. S. Yoon, Comment on "AC frequency characteristics of coplanar impedance sensors as design parameters" by Jongin Hong, Dae Sung Yoon, Sung Kwan Kim, Tae Song Kim, Sanghyo Kim, Eugene Y. Pak and Kwangsoo No, Lab Chip, 2005, 5, 270 (multiple letters), Lab Chip, 2005, 5(12), 1416-1418

32 P. Linderholm, Two-dimensional Microimpedance Imaging for Cell Culture Monitoring (no 3604), EPFL, Lausanne, 2006.

33 H. Morgan and N. G. Green, AC Electrokinetics: Colloids and Nanoparticles, Research Studies Press, Baldock, Hertfordshire, UK, 2002.

\section{Textbooks from the RSC}

The RSC publishes a wide selection of textbooks for chemical science students. From the bestselling Crime Scene to Court, 2nd edition to groundbreaking books such as Nanochemistry: A Chemical Approach to Nanomaterials, to primers on individual topics from our successful Tutorial Chemistry Texts series, we can cater for all of your study needs.

Find out more at www.rsc.org/books

Lecturers can request inspection copies - please contact sales@rsc.org for further information.

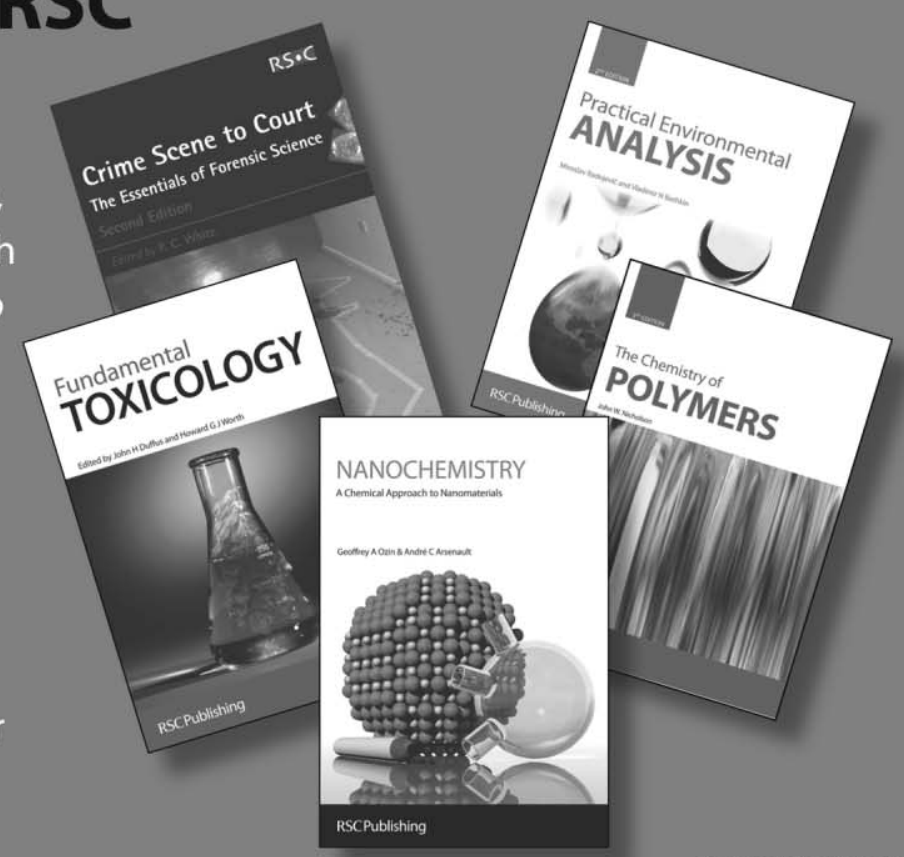

